\title{
Convergence of Discrete Adomian Method for Solving a Class of Nonlinear Fredholm Integral Equations
}

\author{
Ibrahim Lotfy Hassan Alkalla, Reda Abdo Abd-Elmonem, Ayman Mohamed Ali Ahmed Gomaa* \\ Mathematics and Engineering Physics Department, Faculty of Engineering, Mansoura University, Mansoura, Egypt \\ Email:"a_gomaa@mans.edu.eg
}

Received September 25, 2012; revised November 25, 2012; accepted December 3, 2012

\begin{abstract}
In recent papers the solution of nonlinear Fredholm integral equations was discussed using Adomian decomposition method (ADM). For case in which the integrals are analytically impossible, ADM can not be applied. In this paper a discretized version of the ADM is introduced and the proposed version will be called discrete Adomian decomposition method (DADM). An accelerated formula of Adomian polynomials is used in calculations. Based on this formula, a new convergence approach of ADM is introduced. Convergence approach is reliable enough to obtain an explicit formula for the maximum absolute truncated error of the Adomian's series solution. Also, we prove that the solution of nonlinear Fredholm integral equation by DADM converges to ADM solution. Finally, some numerical examples were introduced.
\end{abstract}

Keywords: Nonlinear Fredholm Integral Equations; Contraction Mapping; Adomian Decomposition Method; Quadratures Techniques

\section{Introduction}

Integral equations provide an important tool for modeling a numerous phenomena and processes and also for solving boundary value problems for both ordinary and partial differential equations. Their historical development is closely related to the solution of boundary value problems in potential theory. Progress in the theory of integral equations also had a great impact on the development of functional analysis. Reciprocally, the main results of the theory of compact operators have taken the leading part to the foundation of the existence theory for integral equations of the second kind [1-4]. Therefore, many different methods are used to obtain the solution of the linear and nonlinear integral equations. Among these methods ADM which has gained a great interest in the analytical solutions of linear and nonlinear Fredholm integral equations [5-9]. This is due to many advantages such as simplicity and high accuracy $[5,6]$. The Adomian solution is obtained as an infinite series which converges to exact solution [10], under some mild conditions. In this work, the nonlinear the Fredholm integral equation

$$
\lambda x(t)-\int_{a}^{b} k(t, s) f(x(s)) \mathrm{d} s=y(t), \lambda \neq 0,
$$

is considered where $y(t)$ is known continuous function on $D=[a, b]$ and the kernel $k(t, s)$ is continuous on

${ }^{*}$ Corresponding author. the square $E=\{(t, s): t \in D, s \in D\}$ and bounded such that $|k(t, s)| \leq M$ where, $M$ is the upper bound on the square $E$. The nonlinear term $f(x(s))$ is Lipschitz continuous with $|f(x)-f(z)| \leq L|x-z|, L$ is Lipschitz constant and has Adomian polynomials representation

$$
f(x)=\sum_{n=0}^{\infty} A_{n}\left(x_{0}, x_{1}, \cdots, x_{n}\right),
$$

where the traditional formula of $A_{n}$ is

$$
A_{n}=(1 / n !)\left(\mathrm{d}^{n} / \mathrm{d} \mu^{n}\right)\left[f\left(\sum_{i=0}^{\infty} \mu^{i} x_{i}\right)\right]_{\mu=0} .
$$

The author in $[11,12]$ deduced a new formula to the Adomian's polynomials which can be written in the form

$$
A_{n}=f\left(S_{n}\right)-\sum_{j=0}^{n-1} A_{j}, n \geq 1
$$

where the partial sum $S_{n}=\sum_{i=0}^{n} x_{i}(t)$ and $A_{0}=f\left(x_{0}\right)$. Formula (4) is called an accelerated Adomian polynomials and it was used successfully in [13] for solving a class of nonlinear fractional differential equations and in [14] for solving a class of nonlinear partial differential equations. Formula (4) has the advantage of absence of any derivative terms in the recursion, thereby allowing for ease of computation. In this work, it will be used directly in convergence analysis (see Theorem 2) and all calcula- 
tions concerning the numerical examples. Application of ADM on (1) yields:

$$
x(t)=\sum_{i=0}^{\infty} x_{i}(t),
$$

where the components $x_{i}(t), i \geq 0$ are computed using the following recursive relations

$$
\begin{gathered}
x_{0}(t)=\frac{1}{\lambda} y(t), \\
x_{m+1}(t)=\frac{1}{\lambda} \int_{a}^{b} k(t, s) A_{m}(s) \mathrm{d} s, m \geq 0 .
\end{gathered}
$$

The computation of each component $x_{i}(t), i \geq 1$ requires the computation of integral in Equation (7). If the evaluation of that integral analytically is possible, ADM can be applied in a simple manner. In case where the evaluation of the integral in (7) is analytically impossible, ADM can not be directly applied. In order to overcome this obstacle, please see the details of Sections 2 and 3. In Section 2, a problem is solved in a special case where the kernel $k(t, s)$ is separable [15]. In Section 3, a problem is solved in a more general case where the kernel $k(t, s)$ is not separable and we introduce a discretized modified version of the ADM which is called DADM. In Section 4, convergence of DADM is discussed and the maximum absolute truncated error is estimated. Finally, to verify the theoretical results, some numerical examples are presented in Section 5.

\section{Numerical Implementation of ADM}

For the sake of making this paper self-contained, a brief summary of numerical implementation of ADM will be introduced in this section (for more details see [15]). Let the kernel function be separable of the form

$$
k(t, s)=g_{1}(s) g_{2}(t),
$$

then Equation (7) becomes

$$
x_{m+1}(t)=\frac{1}{\lambda}\left\{g_{2}(t) \int_{a}^{b} g_{1}(s) A_{m}(s) \mathrm{d} s\right\}, m \geq 0 .
$$

Consider any numerical integration scheme to approximate definite integral by the following formula [16-18]

$$
\int_{a}^{b} g(s) \mathrm{d} s \approx \sum_{j=0}^{n} w_{n, j} g\left(s_{n, j}\right),
$$

where $g(s)$ is continuous function on $[a, b]$, $s_{n, j}=a+j h$ are the nodes of the quadrature rule, $h=(b-a) / n$ and $w_{n, j}, j=0,1,2, \cdots, n$ are the weight functions. Applying formula (10) on Equation (9) to obtain

$$
x_{m+1}(t) \approx \frac{1}{\lambda}\left\{g_{2}(t) \sum_{j=0}^{n} w_{n, j} g_{1}\left(s_{n, j}\right) A_{m}\left(s_{n, j}\right)\right\}, m \geq 0 .
$$

Now, the approximate solution of Equation (1) is the sum of all the components $x_{m}(t), m \geq 1$ in Equation (11) and the first component in Equation (6).

\section{Discrete Adomian Decomposition Method}

In case the kernel function $k(t, s)$, is not separable, the integral in (1) can not be computed and hence the ADM will not be able to continue in order to obtain solution. Therefore, we suggest DADM to overcome this obstacle. The idea is to discretize the independent variable; $t$, just before applying the quadrature rule. This gives an opportunity to evaluate the integral in Equation (7) numerically but, of course, at the discretization points of the independent variable. Thus, the discrete version of Equations (6) and (7) may take the form

$$
\begin{gathered}
\tilde{x}_{0}\left(s_{n, i}\right)=\frac{1}{\lambda} y\left(s_{n, i}\right), \text { and } \\
\tilde{x}_{m+1}\left(s_{n, i}\right)=\frac{1}{\lambda} \sum_{j=0}^{n} w_{n, j} k\left(s_{n, i}, s_{n, j}\right) A_{m}\left(s_{n, j}\right), m \geq 0,
\end{gathered}
$$

$s_{n, i}=a+i h, i=0,1, \cdots, n$ and $w_{n, j}$ are the weight functions of any numerical integration scheme. The approximate solution of Equation (1) using DADM can be computed as

$$
\tilde{x}\left(s_{n, i}\right)=\sum_{m=0}^{\infty} \tilde{x}_{m}\left(s_{n, i}\right)
$$

Rewriting Equations (12)-(14) in matrix form

$$
\begin{gathered}
\tilde{X}_{0}=Y, \\
\tilde{X}_{m+1}=B A_{m}, m \geq 0, \text { and } \\
\tilde{X}=\sum_{m=0}^{\infty} \tilde{X}_{m}
\end{gathered}
$$

where $\tilde{X}_{0}, \tilde{X}_{m}, \tilde{X}_{m+1}, \tilde{X}$ and $Y$ are all vectors of dimension $(n+1)$ and $B$ is $(n+1) \times(n+1)$ matrix such that

$$
\begin{aligned}
& \tilde{X}_{0}=\left[\tilde{x}_{0}\left(s_{n, i}\right)\right], i=0,1, \cdots, n, Y=\left[\frac{y\left(s_{n, i}\right)}{\lambda}\right], \\
& i=0,1, \cdots, n, \\
& \tilde{X}_{m}=\left[\tilde{x}_{m}\left(s_{n, i}\right)\right], i=0,1, \cdots, n, A_{m}=\left[\tilde{A}_{m}\left(s_{n, i}\right)\right], \\
& i=0,1, \cdots, n, \text { and } \\
& B=\left[\frac{1}{\lambda} w_{n, j} k\left(s_{n, i}, s_{n, j}\right)\right], i, j=0,1, \cdots, n .
\end{aligned}
$$

The main advantage of the proposed DADM is that the matrix $B$ is unchanged during the computation of components $\tilde{x}_{m}, m \geq 1$ and the computation of the solution need not to solve linear algebraic system of equations like Nystrom method and projection methods. Also, this method can be used for solving Equation (1) with non- 
separable kernel. Thus DADM is more general than the numerical implementation of ADM introduced in [15].

\section{Convergence Approach of DADM}

Convergence of the Adomian series solution was studied for different problems and by many authors. In $[19,20]$ convergence was investigated when the method applied to a general functional equations and to specific type of equations in [21,22]. In convergence analysis, Adomian's polynomials play a very important role however, these polynomials cannot utilize all the information concerning the obtained successive terms of the series solution, which could affect and directly the accuracy as well as the convergence region and the convergence rate. In the present analysis we suggest an alternative approach for proving the convergence. This approach depends mainly on El-Kalla accelerated Adomian polynomial formula (4). As a result to this approach, the maximum absolute truncated error of the series solution is estimated. Define a mapping $F: B \rightarrow B$ where, $B=(C[D],\|\cdot\|)$ is the Banach space of all continuous functions on $D$ with the norm $\|x(t)\|=\max _{\forall t \in D}|x(t)|$.

\subsection{Uniqueness Theorem}

Theorem 1. Problem (1) has a unique solution whenever $0<\alpha<1$, where, $\alpha=\frac{L M(b-a)}{|\lambda|}$

Proof. Define the mapping to be:

$F x(t)=\frac{1}{\lambda} y(t)+\frac{1}{\lambda} \int_{a}^{b} k(t, s) f(x(s)) \mathrm{d} s$. and let $x$ and ${ }^{*}$ be two different solutions to (1) then

$$
\begin{aligned}
\| y-y \mid & =\max _{\forall t \in D}\left|\frac{1}{\lambda} \int_{a}^{b} k(t, s)\left[f(x)-f\left(\begin{array}{c}
* \\
x
\end{array}\right)\right] \mathrm{d} s\right| \\
& \leq \max _{\forall t \in D} \frac{1}{|\lambda|} \int_{0}^{t}|k(t, \tau)|\left|f(y)-f\left(\begin{array}{c}
* \\
y
\end{array}\right)\right| \mathrm{d} \tau \\
& \leq \frac{L M}{|\lambda|}|y-y| \int_{a}^{b} \mathrm{~d} s \leq \alpha|y-y|
\end{aligned}
$$

Under the condition $0<\alpha<1$ the mapping $F$ is contraction therefore, by the Banach fixed-point theorem for contraction [23], there exist a unique solution to problem (1) and this completes the proof.

\subsection{Convergence Theorem}

Theorem 2. The series solution (5) of problem (1) using ADM converges if: $0<\alpha<1$ and $\left|x_{1}\right|<\infty$

Proof. Let $S_{n}$ and $S_{m}$ be arbitrary partial sums with $n \geq m$. We are going to prove that $\left\{S_{n}\right\}$ is a Cauchy sequence in Banach space $B$

$$
\begin{aligned}
\left\|S_{n}-S_{m}\right\| & =\max _{\forall t \in D}\left|S_{n}-S_{m}\right|=\max _{\forall t \in D}\left|\sum_{i=m+1}^{n} x_{i}(t)\right| \\
& =\max _{\forall t \in D}\left|\sum_{i=m+1}^{n} \frac{1}{\lambda} \int_{a}^{b} k(t, s) A_{i-1} \mathrm{~d} s\right| \\
& =\max _{\forall t \in D}\left|\frac{1}{\lambda} \int_{a}^{b} k(t, s) \sum_{i=m}^{n-1} A_{i} \mathrm{~d} s\right| .
\end{aligned}
$$

From Formula (4) we have $\sum_{i=m}^{n-1} A_{i}=f\left(S_{n-1}\right)-f\left(S_{m-1}\right)$

so

$$
\begin{aligned}
& \left\|S_{n}-S_{m}\right\| \\
& =\max _{\forall t \in D}\left|\frac{1}{\lambda} \int_{a}^{b} k(t, s)\left[f\left(S_{n-1}\right)-f\left(S_{m-1}\right)\right] \mathrm{d} s\right| \\
& \leq \max _{\forall t \in D} \frac{1}{|\lambda|} \int_{a}^{b}\left|k(t, s) \| f\left(S_{n-1}\right)-f\left(S_{m-1}\right)\right| \mathrm{d} s \\
& \leq \alpha\left\|S_{n-1}-S_{m-1}\right\| .
\end{aligned}
$$

Let, $n=m+1$ then

$$
\begin{aligned}
& \left\|S_{m+1}-S_{m}\right\| \leq \alpha\left\|S_{m}-S_{m-1}\right\| \leq \alpha^{2}\left\|S_{m-1}-S_{m-2}\right\| \\
& \leq \cdots \leq \alpha^{m}\left\|S_{1}-S_{0}\right\| .
\end{aligned}
$$

From the triangle inequality we have

$$
\begin{aligned}
& \left\|S_{n}-S_{m}\right\| \\
\leq & \left\|S_{m+1}-S_{m}\right\|+\left\|S_{m+2}-S_{m+1}\right\|+\cdots+\left\|S_{n}-S_{n-1}\right\| \\
\leq & {\left[\alpha^{m}+\alpha^{m+1}+\cdots+\alpha^{n-1}\right]\left\|S_{1}-S_{0}\right\| } \\
\leq & \alpha^{m}\left[1+\alpha+\alpha^{2}+\cdots+\alpha^{n-m-1}\right]\left\|S_{1}-S_{0}\right\| \\
\leq & \alpha^{m}\left(\frac{1-\alpha^{n-m}}{1-\alpha}\right)\left\|x_{1}(t)\right\| .
\end{aligned}
$$

Since $0<\alpha<1$ so, $\left(1-\alpha^{n-m}\right)<1$ then

$$
\left\|S_{n}-S_{m}\right\| \leq \frac{\alpha^{m}}{1-\alpha} \max _{\forall t \in D}\left|x_{1}(t)\right| .
$$

But $\left|x_{1}\right|<\infty$ so, as $m \rightarrow \infty$ then $\left\|S_{n}-S_{m}\right\| \rightarrow 0$. We conclude that $\left\{S_{n}\right\}$ is a Cauchy sequence in $C[D]$ so, the series converges and the proof is complete.

\subsection{Error Estimate}

Theorem 3. The maximum absolute truncation error of the series solution (5) to problem (1) is estimated to be:

$$
\begin{aligned}
& \max _{\forall t \in D}\left|x(t)-\sum_{i=0}^{m} x_{i}(t)\right| \leq \frac{K \alpha^{m+1}}{|\lambda| L(1-\alpha)} \text { where } \\
& K=\max _{\forall t \in D}\left|f\left(\frac{1}{\lambda} y(t)\right)\right| .
\end{aligned}
$$

Proof. From Theorem 2 inequality (18) we have 


$$
\left\|S_{n}-S_{m}\right\| \leq \frac{\alpha^{m}}{1-\alpha} \max _{\forall t \in D}\left|x_{1}(t)\right|
$$

As $n \rightarrow \infty$ then $S_{n} \rightarrow x(t)$ and

$$
\begin{aligned}
\max _{\forall t \in D}\left|x_{1}(t)\right| & =\max _{\forall t \in D}\left|\frac{1}{\lambda} \int_{a}^{b} k(t, s) A_{0} \mathrm{~d} s\right| \\
& =\max _{\forall t \in D}\left|\frac{1}{\lambda} \int_{a}^{b} k(t, s) f\left(x_{0}\right) \mathrm{d} s\right| \\
& \leq \frac{1}{|\lambda|} M(b-a) \max _{\forall t \in D}\left|f\left(x_{0}\right)\right|
\end{aligned}
$$

so,

$$
\left\|x(t)-S_{m}\right\| \leq \frac{\alpha^{m+1}}{|\lambda| L(1-\alpha)} \max _{\forall t \in D}\left|f\left(\frac{1}{\lambda} y(t)\right)\right| .
$$

Finally, the maximum absolute truncation error in the interval $D$ is:

$$
\max _{\forall t \in D}\left|x(t)-\sum_{i=0}^{m} x_{i}(t)\right| \leq \frac{K \alpha^{m+1}}{|\lambda| L(1-\alpha)} .
$$

This completes the proof.

\subsection{Equivalence between DADM and ADM}

Let $D$ be a closed bounded set in $\boldsymbol{R}^{l}, l \geq 1$, and define operator $\kappa$ such that

$$
\kappa f(x(t))=\int_{D} k(t, s) f(x(s)) \mathrm{d} s, t \in D, x \in C(D),
$$

where $\kappa$ is a compact operator on $C(D)$ to $C(D)$ and is bounded on $C(D)$ to $C(D)$ since

$$
\|\kappa f(x(t))\| \leq\|\kappa\| \cdot\|f(x(t))\| \text { and }\|\kappa\|=\max _{t \in D} \int_{D}|k(t, s)| \mathrm{d} s \text {. }
$$

Now, Equation (1) can be written as

$$
\lambda x-\kappa f(x)=y,
$$

let $x$ be the solution obtained by using ADM, where $x(t)=\sum_{m=0}^{\infty} x_{m}(t)$, and $x_{0}=y / \lambda$. Define numerical integral operator $\kappa_{n}$ as

$$
\kappa_{n} f(\tilde{x}(t))=\sum_{j=0}^{n} w_{n, j} k\left(t, s_{n, j}\right) f\left(\tilde{x}\left(s_{n, j}\right)\right),
$$

where $\kappa_{n}$ is linear finite rank bounded operator on $C(D)$ to $C(D)$ since

$$
\left\|\kappa_{n}\right\|=\max _{t \in D} \sum_{j=0}^{n}\left|w_{n, j} k\left(t, s_{n, j}\right)\right| .
$$

With the operator $\kappa_{n}$, Equation (1) may be written as

$$
\lambda \tilde{x}-\kappa_{n} f(\tilde{x})=y,
$$

where $\tilde{x}$ here is the solution obtained by using DADM, and $\tilde{x}(t)=\sum_{m=0}^{\infty} \tilde{x}_{m}(t)$, and $\tilde{x}_{0}=y / \lambda$.

Theorem 4. Since $\left\|\kappa_{n} g-\kappa g\right\| \rightarrow 0$ as $n \rightarrow \infty$ where $g \in C(D)$ [18]. Then, the solution of Equation (1), using DADM converges to the solution of the same equation when using $\mathrm{ADM}$, i.e.

$$
\tilde{x} \rightarrow x \text { as } n \rightarrow \infty,
$$

Proof. Since

$x(t)=\sum_{m=0}^{\infty} x_{m}(t),, x_{0}=\frac{y}{\lambda}, \tilde{x}(t)=\sum_{m=0}^{\infty} \tilde{x}_{m}(t)$, and $\tilde{x}_{0}=\frac{y}{\lambda}$.

Starting with

$$
\|\tilde{X}-x\|=\left\|\sum_{m=0}^{\infty}\left(\tilde{x}_{m}-x_{m}\right)\right\| \leq \sum_{m=0}^{\infty}\left\|\tilde{x}_{m}-x_{m}\right\| .
$$

Since

$$
\begin{gathered}
\left\|\tilde{x}_{0}-x_{0}\right\|=\left\|\frac{y}{\lambda}-\frac{y}{\lambda}\right\|=0, \text { and } \\
\left\|\tilde{x}_{m}-x_{m}\right\|=\left\|\frac{1}{\lambda} \kappa_{n} A_{m-1}-\frac{1}{\lambda} \kappa A_{m-1}\right\| \\
=\frac{1}{\lambda}\left\|\kappa_{n} A_{m-1}-\kappa A_{m-1}\right\| \rightarrow 0 \text { as } n \rightarrow \infty
\end{gathered}
$$

Then, by induction and substituting from Equation (25) and Equation (26) into inequality (24), this completes the proof.

\section{Numerical Experiments}

Consider the following linear Fredholm integral equation

$$
\begin{aligned}
& x(t)-\int_{0}^{1} \frac{\exp \left(t^{2}+s^{2}\right)}{10} x(s) \mathrm{d} s \\
= & t-\frac{1}{20}\left\{\exp \left(t^{2}+1\right)-\exp \left(t^{2}\right)\right\}
\end{aligned}
$$

whose exact solution is $x(t)=t$. In this example the ADM can not be applied because the evaluation of $x_{m}(t), m \geq 1$ is conditioned to compute $\int_{0}^{1} \exp \left(s^{2}\right) \mathrm{d} s$. Since, the kernel is separable, the numerical implementation of ADM introduced in [15] can be used as well as DADM.

The solution by numerical implementation of ADM introduced in [15]

$$
x_{0}(t)=t-\frac{1}{20}\left\{\exp \left(t^{2}+1\right)-\exp \left(t^{2}\right)\right\},
$$

and the computation of $x_{m}(t), m \geq 1$ needs Equation (11) and Simpson's rule [16-18] with number of subintervals $n=10$, and step size $h=\frac{1}{10}$ to obtain 


$$
\begin{aligned}
& x_{1}(t)=\frac{\exp \left(t^{2}\right)}{10} \int_{0}^{1} \exp \left(s^{2}\right) x_{0}(s) \mathrm{d} s=0.065602 \exp \left(t^{2}\right), \\
& x_{2}(t)=\frac{\exp \left(t^{2}\right)}{10} \int_{0}^{1} \exp \left(s^{2}\right) x_{1}(s) \mathrm{d} s \simeq 0.015514 \cos \left(t^{2}\right),
\end{aligned}
$$

and so on. The approximate solution by this method is

$$
\stackrel{*}{x}_{15}(t)=\sum_{m=0}^{15} x_{m}(t)=t+6.9255 \times 10^{-6} \exp \left(t^{2}\right),
$$

and the maximum error is

$$
\left\|E_{15}(t)\right\|_{\max }=1.8825 \times 10^{-5}
$$

Using Equations (15)-(17) and Simpson's rule with number of sub-intervals $n$ and step size $h=1 / n$, the results of DADM can be tabulated in Table 1. Table 1 shows the effect of $n$ and $m$ in the maximum absolute error $\left|e_{r}(t)\right|_{\max }=\left|x(t)-{ }^{*} x_{r}(t)\right|_{\max }$.

Example (2) consider the following nonlinear Fredholm integral equation

$$
\begin{aligned}
& x(t)-\int_{0}^{1} \frac{1}{10} \exp \left(s^{4}+t^{3}\right)[x(s)]^{3} \mathrm{~d} s \\
= & t-\frac{1}{40}\left[\exp \left(t^{3}+1\right)-\exp \left(t^{3}\right)\right]
\end{aligned}
$$

whose exact solution is $x(t)=t$. In this example the ADM can not be applied because the integral

$\int_{0}^{1} \exp \left(s^{4}\right) \mathrm{d} s$ has no analytical solution. The numerical implementation of ADM introduced by [15] can be used, because the kernel is separable. Also, DADM can be used to obtain solution. Table 2 shows the effect of $n$ and $m$ in the maximum absolute error

$$
\left|e_{r}(t)\right|_{\text {max }}=\left|x(t)-{ }^{*} x_{r}(t)\right|_{\max } .
$$

Example (3) consider the following nonlinear Fredholm integral equation

$$
\begin{aligned}
& x(t)-\int_{0}^{1} \frac{1}{10} \cos [\exp (s)+t] \exp (x(s)) \mathrm{d} s \\
= & t-\frac{1}{10}[\sin (t+\exp (1))-\sin (t+1)]
\end{aligned}
$$

whose exact solution is $x(t)=t$. In this example the ADM can not be applied because the integral

$\int_{0}^{1} \cos [\exp (s)+t] \exp [\sin (s)] \mathrm{d} s$ has no analytical solution. Also, the numerical implementation of ADM introduced by [15] can not be used, because the kernel function is not separable. Here, DADM is the suitable method to obtain solution. Table 3 shows the effect of $n$ and $m$ in
Table 1. The effect of $\boldsymbol{n}$ and $\boldsymbol{m}$ in the maximum absolute error (example 1).

\begin{tabular}{cc}
\hline$n=4, m=5$ & Maximum absolute error \\
\hline$n=8, m=7$ & $8.7310 e^{-0.05}$ \\
$n=18, m=9$ & $4.4872 e^{-0.06}$ \\
$n=24, m=10$ & $3.8303 e^{-0.08}$ \\
$n=48, m=12$ & $6.0570 e^{-0.09}$ \\
$n=50, m=12$ & $6.0346 e^{-0.10}$ \\
\hline
\end{tabular}

Table 2. The effect of $n$ and $m$ in the maximum absolute error (example 2).

\begin{tabular}{cc}
\hline & Maximum absolute error \\
\hline$n=4, m=3$ & $3.2539 e-0.03$ \\
$n=8, m=4$ & $2.0713 e-0.04$ \\
$n=10, m=5$ & $3.2843 e-0.05$ \\
$n=14, m=6$ & $4.6748 e-0.06$ \\
$n=26, m=8$ & $3.5334 e-0.07$ \\
$n=36, m=9$ & $6.8715 e-0.08$ \\
\hline
\end{tabular}

Table 3. the effect of $\boldsymbol{n}$ and $\boldsymbol{m}$ in the maximum absolute error (example 3).

\begin{tabular}{cc}
\hline & Maximum absolute error \\
\hline$n=2, m=3$ & $3.0428 e-0.03$ \\
$n=4, m=3$ & $1.1742 e-0.04$ \\
$n=6, m=5$ & $4.4983 e-0.05$ \\
$n=10, m=5$ & $4.6254 e-0.06$ \\
$n=20, m=7$ & $3.6948 e-0.07$ \\
$n=34, m=7$ & $2.4078 e-0.08$ \\
\hline
\end{tabular}

the maximum absolute error $\left|e_{r}(t)\right|_{\max }=\left|x(t)-x_{r}(t)\right|_{\max }$.

\section{Conclusion}

Based on the accelerated Adomian polynomials formula (4) and the well known contraction mapping principles, convergence of DADM is discussed. Convergence approach is reliable enough to obtain an explicit formula for the maximum absolute truncated error of the Adomian's series solution. The proposed DADM is more general method than that in [15] because it is capable to solve linear and nonlinear Fredholm integral equation with separable as well as non-separable kernel functions. DADM is recommended to solve linear and nonlinear Fredholm integral equation due to many advantages such 
as the matrix $B$ is unchanged during the computation of the components, the solution need not to solve linear algebraic system of equations like Nystrom method and projection methods. Another advantage when applying DADM to solve linear Fredholm integral equation with symmetric kernel $k(t, s)=k(s, t)$ is the matrix $B$ will be symmetric matrix as in example (1).

\section{REFERENCES}

[1] K. E. Atkinson, "A Survey of Numerical Methods for the Solution of Fredholm Integral Equations of the Second Kind," Society for Industrial and Applied Mathematics, Philadelphia, 1976, p. 237.

[2] F. G. Tricomi, "Integral Equations," Dover Publications Inc., New York, 1985.

[3] L. M. Delves and J. L. Mohamed, "Computational Methods for Integral Equations," Cambridge University Press, New York, 1985. doi:10.1017/CBO9780511569609

[4] M. A. Golbluerg, "Numerical Solution of Integral Equations," Plenum Press, New York, 1990.

[5] G. Adomian, "Solving Frontier Problems of Physics: The Decomposition Method," Kluwer Academic Publishers, Dordrecht, 1994.

[6] A. M. Wazwaz and S. A. Khuri, "Two Methods for Solving Integral Equations," Applied Mathematics and Computation, Vol. 77, No. 1, 1996, pp. 79-89. doi:10.1016/0096-3003(95)00189-1

[7] A. M. Wazwaz, "A First Course in Integral Equations," World Scientific Publishing Co., Singapore City, 1997. doi:10.1142/3444

[8] E. Babolian, J. Biazar and A. R. Vahidi, "The Decomposition Method Applied to Systems of Fredholm Integral Equations of the Second Kind," Applied Mathematics and Computation, Vol. 148, No. 2, 2004, pp. 443-452. doi:10.1016/S0096-3003(02)00859-7

[9] A. M. Wazwaz, "A Comparison Study between the Modified Decomposition Method and the Traditional Methods for Solving Nonlinear Integral Equations," Applied Mathematics and Computation, Vol. 181, No. 2, 2006, pp. 1703-1712. doi:10.1016/j.amc.2006.03.023

[10] Y. Cherruault, G. Adomian, K. Abbaoui and R. Rach, "Further Remarks on Convergence of Decomposition Method," International Journal of Bio-Medical Computing, Vol. 38, No. 1, 1995, pp. 89-93. doi:10.1016/0020-7101(94)01042-Y

[11] I. L. El-kalla, “Convergence of Adomian's Method Applied to a Class of Volterra Type Integro-Differential
Equations," International Journal of Differential Equations and Applications, Vol. 10, No. 2, 2005, pp. 225-234.

[12] I. L. El-kalla, "Error Analysis of Adomian Series Solution to a Class of Nonlinear Differential Equations," Applied Mathematics E-Notes, Vol. 7, 2007, pp. 214-221.

[13] I. L. El-Kalla, "Error Estimate of the Series Solution to a Class of Nonlinear Fractional Differential Equations," Communications in Nonlinear Science and Numerical Simulation, Vol. 16, No. 3, 2011, pp. 1408-1413. doi:10.1016/j.cnsns.2010.05.030

[14] E. A. Az-Zo'bi and K. Al-Khaled, "A New Convergence Proof of the Adomian Decomposition Method for a Mixed Hyperbolic Elliptic System of Conservation Laws," Applied Mathematics and Computation, Vol. 217, No. 8, 2010, pp. 4248-4256.

[15] E. Babolian and A. Davari, "Numerical Implementation of Adomain Decomposition Method," Applied Mathematics and Computation, Vol. 153, No. 1, 2004, pp. 301305. doi:10.1016/S0096-3003(03)00646-5

[16] A. J. Jerri, "Introduction to Integral Equations with Applications," John Wiley \& Sons Inc., New York, 1999.

[17] J. Stoer and R. Bulirsch, "Introduction to Numerical Analysis," 3rd Edition, Springer-Verlag, Berlin, Heidelberg, New York, 2002.

[18] K. E. Atkinson, "The Numerical Solution of Integral Equations of the Second Kind," Cambridge University Press, New York, 1997. doi:10.1017/CBO9780511626340

[19] K. Abbaoui and Y. Cherruault, "New Ideas for Proving Convergence of Decomposition Methods," Computers \& Mathematics with Applications, Vol. 29, No. 7, 1995, pp. 103-108. doi:10.1016/0898-1221(95)00022-Q

[20] M. M. Hosseini and H. Nasabzadeh, "On the Convergence of Adomian Decomposition Method," Applied Mathematics and Computation, Vol. 182, No. 1, 2006, pp. 536-543. doi:10.1016/j.amc.2006.04.015

[21] K. Abbaoui and Y. Cherruault, "Convergence of Adomian's Method Applied to Differential Equations," Computers \& Mathematics with Applications, Vol. 28, No. 5, 1994, pp. 103-109. doi:10.1016/0898-1221(94)00144-8

[22] R. Rajaram and M. Najafi, "Analytical Treatment and Convergence of the Adomian Decomposition Method for a System of Coupled Damped Wave Equations," Applied Mathematics and Computation, Vol. 212, No. 1, 2009, pp. 72-81. doi:10.1016/j.amc.2009.02.006

[23] E. Kreyszig, "Introductory Functional Analysis with Applications,” John Wiley \& Sons Inc., New York, 1978. 